\title{
LXXXI. Pendulum motion and spherical trigonometry
}

\section{G. Greenhill}

To cite this article: G. Greenhill (1910) LXXXI. Pendulum motion and spherical trigonometry, Philosophical Magazine Series 6, 20:118, 728-740, DOI: 10.1080/14786441008636960

To link to this article: http://dx.doi.org/10.1080/14786441008636960

曲 Published online: 21 Apr 2009.

Submit your article to this journal ๘

Џll Article views: 2

Q View related articles $\sqsubset$ 
It will be seen that fused silica has qualities which commend it for use as a material for standards of length. A silica standard metre is on the point of completion at the National Physical Laboratory, and there is good reason to believe that its adoption will be attended with success.

The National Physical Laboratory, Teddington.

\section{Pendulum Motion and Spherical Trigunometry.} By G. Greenhill *

MR. ROSE-INNES has developed the relation between 1 the revolution of a pendulum in a plane and the projection of the motion on a spherical surface, and he shows that the argument of the elliptic function required can be represented by an area on the sphere which grows uniformly with the time (Phil. Mag. June 1910).

In a change to the polar reciprocal, the time will then be represented by a spherical arc, as discussed here in $\S 9$.

1. Consider a circle $A Q D$ on the vertical diameter $A D$, and a particle $\mathrm{Q}$ circulating round it under gravity with velocity due to the depth $\mathrm{KQ}$ below a horizontal line $\mathrm{HK}$; the motion of $Q$ will represent a pendulum making complete revclutions, like a bicycle-wheel on its ball-bearings, put out of balance by an iron bar in the spokes (fig. 1).

The lettering and notation is that employed in my 'Elliptic Functions,' fig. 13, where, with $\mathrm{ADQ}=\phi$,

$$
\begin{aligned}
& \text { (1) } \begin{aligned}
\mathrm{KQ}=\mathrm{AE}-\mathrm{AN}=\mathrm{AE}-\mathrm{AD} \sin ^{2} \phi \\
=\mathrm{AE}\left(1-\kappa^{2} \sin ^{2} \phi\right)=\mathrm{AE} \cdot \Delta^{2} \phi, \kappa^{2}=\frac{\mathrm{AD}}{\mathrm{AE}},
\end{aligned} \\
& \text { (2) (vel. of } \mathrm{Q})^{2}=\left(\mathrm{AD} \frac{d \phi}{d t}\right)^{2}=2 g \cdot \mathrm{KP} \\
& =2 g \cdot \mathrm{AE} \cdot \Delta^{2} \phi,\left(\frac{d \phi}{d t}\right)^{2}=\frac{g}{\mathrm{AC}} \cdot \frac{\Delta^{2} \phi}{\kappa^{2}}, \\
& \text { (3) } \int_{0} \frac{d \phi}{\Delta \phi}=\frac{n t}{\kappa}=u, \quad \phi=\mathrm{am} u, \quad n=\sqrt{\frac{g}{\mathrm{AC}} ;}
\end{aligned}
$$

so that $n / \pi$ is the number of beats per second in small oscillation; and the elliptic argument $u$ grows uniformly with the time $t$, starting from the lowest point $A$.

Draw the circle, centre $\mathrm{E}$ and radius $\mathrm{EB}$, orthogonal to

* Communicated by the Author. 
Motion and Spherical Trigonometry.

Fig. 1.

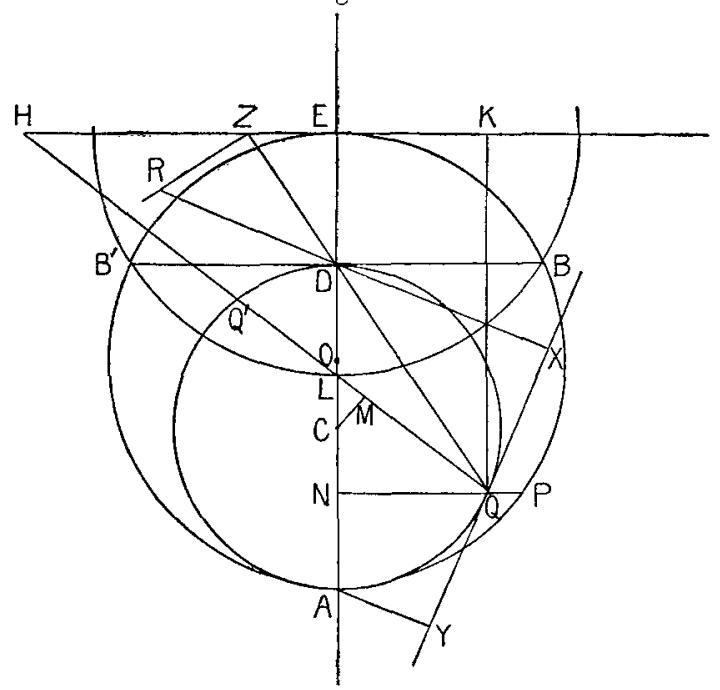

the circle $\mathrm{AQD}$, cutting $\mathrm{AD}$ in $\mathrm{L}$, the limiting point or Landen point ; then

(4)

$$
\text { (4) } \begin{aligned}
& \mathrm{ED} . \mathrm{EA}=\mathrm{EL}^{2} \text {, and } \mathrm{HQ} \cdot \mathrm{HQ}^{\prime}=\mathrm{HL}^{2} \text {, } \\
& \mathrm{QL}^{2}=(\mathrm{HQ}-\mathrm{HL})^{2}=\mathrm{HQ}^{2}-2 \mathrm{HQ} \cdot \mathrm{HL}+\mathrm{HQ} \cdot \mathrm{HQ}^{\prime} \\
&=\left(\mathrm{HQ}+\mathrm{HQ}^{\prime}-2 \mathrm{HL}\right) \mathrm{HQ}=(2 \mathrm{HM}-2 \mathrm{HL}) \mathrm{HQ} \\
&=2 \mathrm{LM} \cdot \mathrm{HQ}=2 \mathrm{LC} \cdot \mathrm{KQ} \\
&=2 \mathrm{TC} \cdot \mathrm{AE} \cdot \Delta^{2} \phi=\mathrm{AL}^{2} \cdot \Delta^{2} \phi .
\end{aligned}
$$

Fig. 2.

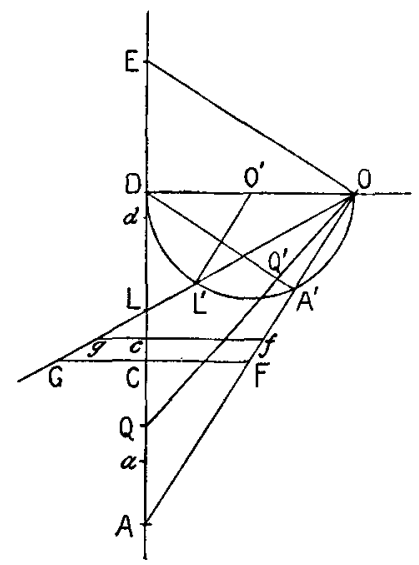

2. Turn the vertical circle about the diameter $A D$ through a right angle, so as to bring DB to DO in fig. 2, and project 
in Mr. Rose-Innes's manner on a sphere, by lines drawn from the centre $O$; corresponding points on the sphere in fig. 4 and the plane AQD of fig. 1 may be represented without confusion by the same letter, in most cases. Then

$$
\kappa^{2}=\frac{\mathrm{AD}}{\mathrm{AE}}=\frac{\mathrm{AD}^{2}}{\mathrm{AO}^{2}}=\sin ^{2} \mathrm{AOD} ;
$$

so that $A O D$ is the modular angle, denoted by $c$; and $\mathrm{EO}=\mathrm{EL}$, and $\mathrm{OL}$ bisects the angle $\mathrm{AOD}$.

Invert with respect to $O$, making $O Q \cdot O Q^{\prime}=O D^{2}$; the Fig. 3.

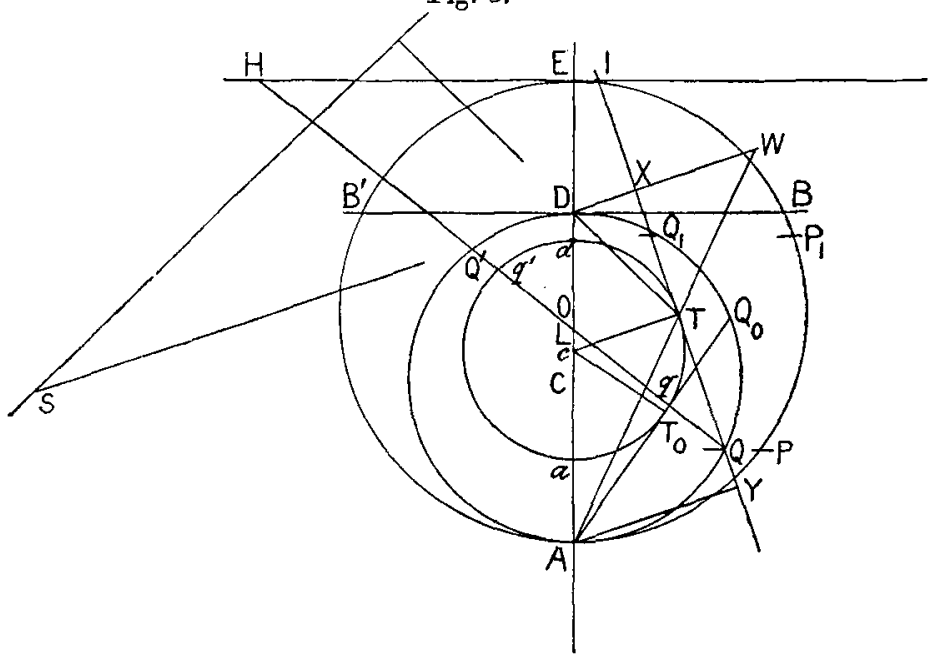

vertical plane $A Q D$ inverts into a sphere on the diameter $O D$, and the circle $A Q D$ into another circle $A^{\prime} Q^{\prime} D$ in a plane perpendicular to $\mathrm{OA}$, so that these circles are circular sections of the cone, vertex $O$ and base $A Q D$.

Now $A Q$ is perpendicular to the plane $O D Q$, so that the planes $O A Q, O D Q$ are at right angles, and the angle $A Q D$ on the sphere in fig. 4 is a right angle.

If $\mathrm{DX}$ is the perpendicular on the tangent at $\mathrm{Q}, \mathrm{QDX}=$ $\mathrm{QDA}=\phi$ in figs. 1 and 4 ; so also in fig. 4 , if $A Y$ is the perpendicular from $A$ on the tangent at $Q, Q A Y=Q A D=\phi^{\prime}$, but $\phi^{\prime}$ is the angle $Q^{\prime} A^{\prime} D$ in fig. 2, or $A D Q^{\prime}$ in fig. 1 .

3. In fig. 4, by Spherical Trigonometry,

(1) $\sin \mathrm{AQ}=\sin \mathrm{AD} \sin \mathrm{ADQ}=\kappa \sin \phi, \cos \mathrm{AQ}=\Delta \phi$,

(2) $\sin \mathrm{DQ}=\boldsymbol{\kappa} \sin \phi^{\prime}, \quad \cos \mathrm{DQ}=\Delta \phi^{\prime}$,

(3) $\cos A Q \cos \mathrm{DQ}=\cos \mathrm{AD}=\cos c, \Delta \phi \Delta \phi^{\prime}=\kappa^{\prime}$,

(4) $\phi^{\prime}=\operatorname{am}(\mathrm{K}-u)$, with $\phi=\operatorname{am} u$. 
Fig. 4.

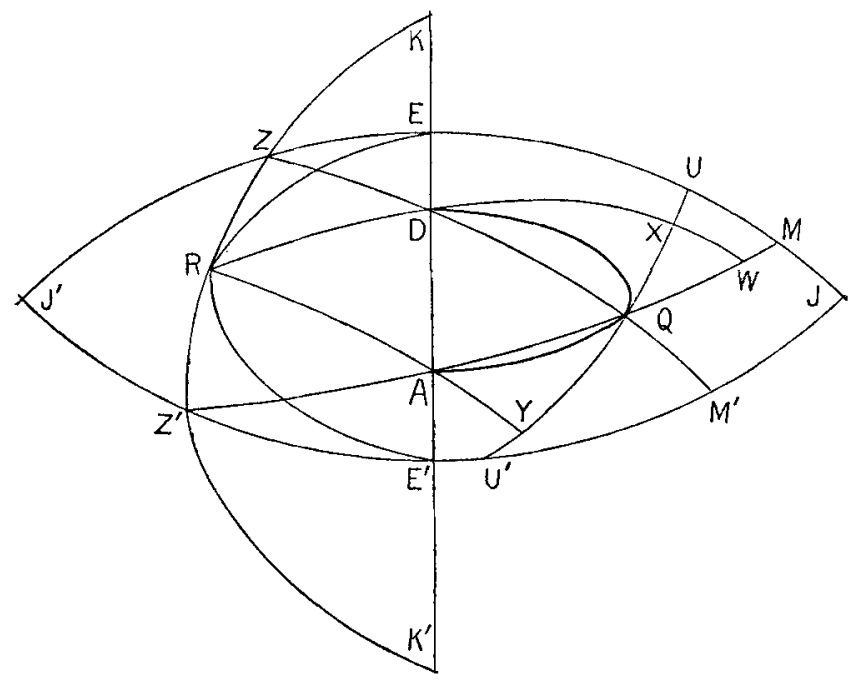

With EJ the polar circle of $A$, and $E^{\prime} J$ of $D$, and from (3) $\S 1$, when $Q$ makes a small advance to $q$, and $M, M^{\prime}$ to $m, m^{\prime}$,

(5) $\quad d u=\frac{d \phi}{\Delta \phi}=\frac{\Delta \phi^{\prime} d \phi}{\kappa^{\prime}}=\frac{\cos D Q}{\cos c} d \phi$

$$
=\frac{\text { spherical area } Q M^{\prime} m^{\prime} q}{\cos c},
$$

(6) $-d u=\frac{d \phi^{\prime}}{\Delta \phi^{\prime}}=\frac{\Delta \phi d \phi^{\prime}}{\kappa^{\prime}}=\frac{\cos \Delta Q}{\cos c} d \phi^{\prime}$

$$
=\frac{\text { spherical area } \mathrm{QM} m q}{\cos c},
$$

$$
u=\frac{\operatorname{area~} \mathrm{AE}^{\prime} \mathrm{M}^{\prime} \mathrm{Q}}{\cos c}, \mathrm{~K}-u=\frac{\operatorname{area} \mathrm{DEMQ}}{\cos c}
$$

(8) $\mathrm{K}=\frac{\operatorname{area} \mathrm{AE}^{\prime} \mathrm{JDQA}}{\cos c}$.

The point $Q$ describes a sphero-conic, with $E J, E^{\prime} J$ the cyclic ares, since

(9) $\quad \cos \mathrm{AQ} \cos \mathrm{DQ}=\sin \mathrm{QM} \sin \mathrm{QM}^{\prime}=\cos c$,

(Salmon, 'Solid Geometry,' Chap. X.), and the tangent $\mathrm{UQU}^{\prime}$ intercepted by the cyclic ares is bisected at $Q$, and 
cuts off a constant area $\mathrm{UJU}^{\prime}=\pi-2 c$; so that the angles $J U^{\prime}, J^{\prime} U^{\prime}$ are equal to the angle $\mathrm{UJU}^{\prime}$, and then

$$
\mathrm{JQ}=\mathrm{QU}=\mathrm{QU}^{\prime}, \mathrm{JM}=\mathrm{MU}, \mathrm{JM}^{\prime}=\mathrm{M}^{\prime} \mathrm{U}^{\prime} \text {. }
$$

Since $A U$ is a quadrant and $A Y U$ a right angle, $Y U$ is a quadrant, and so also is $\mathrm{XU}^{\prime}$; and $\mathrm{XU}^{\prime}=\mathrm{YU}, \mathrm{QX}=\mathrm{QY}$.

If $\mathrm{DX}$ cuts $\mathrm{AM}$ in $W$, the spherical triangles $\mathrm{XQW}$, YQA are equal, and

$$
\begin{aligned}
& \mathrm{DWQ}=\mathrm{QAY}=\mathrm{DAQ}=\phi^{\prime}, \quad \mathrm{DW}=\mathrm{DA}, \\
& \mathrm{DW}=\mathrm{DX}+\mathrm{XW}=\mathrm{DX}+\mathrm{AY}=\mathrm{DA}=c .
\end{aligned}
$$

4. So much for the geometry of the sphero-conic AQD, as developed in Salmon's 'Solid Geometry' and by Mr. RoseInnes; returning to the vertical circle $A Q D$, draw another interior circle aqd, centre $c$, with the same limiting point $\mathrm{L}$ and radical axis $\mathrm{HEK}$, cutting $\mathrm{QLQ}^{\prime}$ in $q, q^{\prime}$; then (fig. 3)

(1) $\mathrm{H} q \cdot \mathrm{H}^{\prime}=\mathrm{HL}^{2}=\mathrm{HQ} \cdot \mathrm{HQ}^{\prime}$,

$$
\begin{aligned}
\mathrm{Q} q \cdot \mathrm{Q} q^{\prime} & =(\mathrm{HQ}-\mathrm{H} q)\left(\mathrm{HQ}-\mathrm{H}_{q^{\prime}}\right) \\
& =\mathrm{HQ}^{2}-\left(\mathrm{H} q+\mathrm{H} q^{\prime}\right) \mathrm{HQ}+\mathrm{HQ} \cdot \mathrm{HQ}^{\prime} \\
& =\left(\mathrm{HQ}+\mathrm{HQ}-\mathrm{H} q-\mathrm{H} q^{\prime}\right) \mathrm{HQ} \\
& =2 \mathrm{M} m \cdot \mathrm{HQ}=2 \mathrm{C} c \cdot \mathrm{KQ} .
\end{aligned}
$$

If $\mathrm{QT}$ is the tangent to this inner circle, cutting the outer circle again in $Q_{1}$,

$$
\begin{aligned}
& \mathrm{QT}^{2}=2 \mathrm{C} c \cdot \mathrm{KQ}, \quad \mathrm{QL}^{2}=2 \mathrm{CL} \cdot \mathrm{KQ}, \\
& \mathrm{QT}^{2}=\frac{\mathrm{C} c}{\mathrm{CL}}=\frac{\mathrm{Q}_{1} \mathrm{~T}^{2}}{\mathrm{Q}_{1} \mathrm{~L}^{2}},
\end{aligned}
$$

and $L T$ bisects the angle $Q L Q_{1}$; also $I T=I L$, if $Q_{1}$ cuts $\mathrm{HE}$ in I, giving a simple construction of the inner circle for a given $Q_{Q_{1}}$.

As the tangent $\mathrm{QTQ}_{1}$ moves round, cutting the outer circle at equal angies,

$$
\frac{\text { vel. of } Q_{1}}{\text { vel. of } Q}=\frac{Q_{1} T}{Q^{T}}=\sqrt{\frac{K_{1} Q_{1}}{K Q}},
$$

and this is the ratio of the velocity under gravity of two particles, $Q$ and $Q_{1}$, describing the circle in the same manner, so that $Q$ and $Q_{1}$ will remain simultaneous positions of the particles if $Q Q_{1}$ is a tangent of the inner circle ; and putting $\mathrm{ADQ}_{1}=\psi=\operatorname{am} u_{1}$, then

$$
u_{1}-u=v, \text { a constant. }
$$

5. Draw gcf in fig. 2 through $c$ perpendicular to the plane $\mathrm{AQD}$, cutting OA in $f$, and $O \mathrm{~L}$ in $g$; the circle, centre $f$ and 
radius $f O$ will pass through $a$ and $d$, since

(1) $\mathrm{E} a \cdot \mathrm{E} d=\mathrm{EL}^{2}=\mathrm{EO}^{2}$, and

(2) $a \mathrm{OL}=\mathrm{EOL}-\mathrm{EO} a=\mathrm{ELO}-\mathrm{E} d \mathrm{O}=d \mathrm{OL}$,

so that $\mathrm{OL}$ bisects the angle $a \mathrm{O} d$, and passes through $g$ on the circle round Oad.

Then $g \mathrm{~L} a, g a \mathrm{O}$ are similar triangles, and $g \mathrm{~L} . g \mathrm{O}=g a^{2}$, so that $\mathrm{L}$ and $\mathrm{O}$ are inverse points with respect to the sphere, centre $g$ and radius $g a$; thence $\mathrm{LT}: \mathrm{TO}$ is a constant ratio, equal to $\mathrm{L} a: a \mathrm{O}$, and similarly $\mathrm{LQ}: \mathrm{QO}$ is the constant ratio $\mathrm{LA}: \mathrm{AO}$ round the circle $\mathrm{AQD}$, and $O Q=O A \cdot \Delta \phi$.

The inverse of the circle $a \mathrm{~T} d$ with respect to $\mathrm{O}$ is another circle $a^{\prime} \mathrm{T}^{\prime} d^{\prime}$ parallel to $\mathrm{A}^{\prime} \mathrm{Q}^{\prime} \mathrm{D}$; for $\mathrm{L}^{\prime} \mathrm{T}^{\prime}$ : $\mathrm{OL}=\mathrm{L} \mathrm{T}: \mathrm{OT}$, a constant ratio, so that $\mathrm{L}^{\prime} \mathrm{T}^{\prime}$ is constant, $\mathrm{L}^{\prime}$ being the point inverse to $L$.

Conversely the inverse of a system of parallels of latitude on a sphere with respect to a point $O$ on the sphere is a system of dipolar circles in a plane, as the circles of latitude on the stereographic representation of a hemisphere.

6 . The line $\mathrm{O}^{\prime} \mathrm{T}^{\prime}$ from $O^{\prime}$, the centre of the sphere on the diameter $O D$, makes a constant angle, $c^{\prime}$, with $O^{\prime} \mathrm{L}^{\prime}$, and the angle $\mathrm{DO}^{\prime} \mathrm{T}^{\prime}$ is double the angle $\mathrm{DOT}^{\prime}$; so that if the are DT in fig. 5 in the representation on a sphere, centre $O$, is produced to double length to $\mathrm{V}, \mathrm{OV}$ will make a constant angle $e^{\prime}$ with $\mathrm{OA}$, which is parallel to $\mathrm{O}^{\prime} \mathrm{L}^{\prime}$, and the arc $\mathrm{AV}=c^{\prime}$.

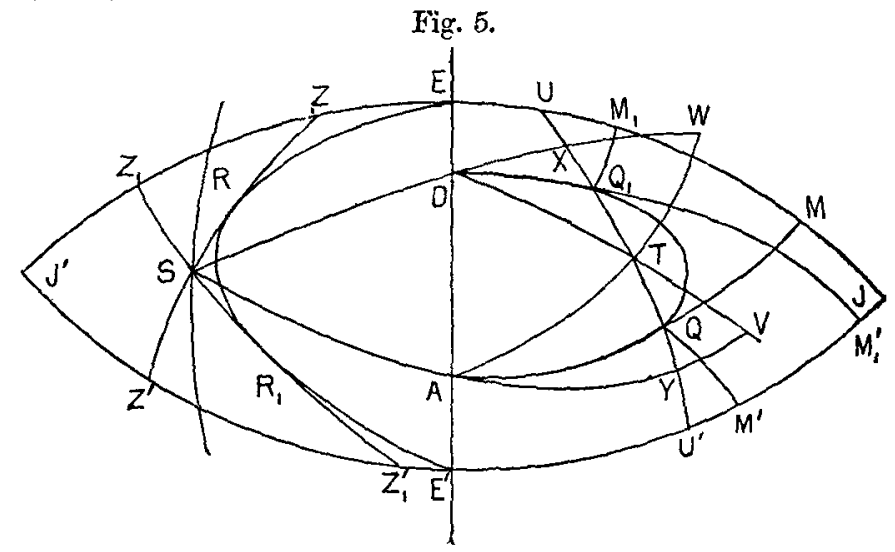

Then in fig. 5, by Spherical Trigonometry,

(1) $\cos \mathrm{AT}^{\mathrm{T}} \cdot \cos \mathrm{DT}=\frac{1}{2}(\cos \mathrm{AD}+\cos \mathrm{AV})=\frac{1}{2}\left(\cos c+\cos c^{\prime}\right)$, a constant; so that $\mathrm{T}$ describes another sphero-conic, interior 
to that described by $Q$, and with the same cyclic arcs, so that its tangent $\mathrm{QQ}_{1}$ cuts off from the cyclic ares a triangle $\mathrm{UJU}^{\prime}$ of constant area, and $\mathrm{UU}^{\prime}$ is bisected at $\mathrm{T}$ (Salmon, 'Solid Geometry,' $\$ 247,248$ ).

But since, in figs. 2, 3,

$$
\frac{\mathrm{QT}}{\mathrm{TQ}}=\frac{\mathrm{QL}}{\mathrm{LQ} \mathrm{Q}_{1}}=\frac{\mathrm{QO}}{\mathrm{OQ}},
$$

OT bisects the angle $Q O Q_{1}$, and T in fig. 5 is the midpoint of $\mathrm{QQ}_{1}$, so that (Salmon, §252) $\mathrm{QQ}_{1}$ cuts off a constant area from the outer sphero-conic.

With constant $u_{1}-u=w$, the area $\mathrm{QMM}_{1} \mathrm{Q}_{1}$ is constant, so that the spherical quadrilateral $Q M M_{1} Q_{1}$ is constant, and this implies that the sum of the angles $D Q Q_{1}, D Q_{1} Q$ or $D Q V$ is constant, and this is found in $\$ 8$ to be $\operatorname{am}(K-w)+\frac{1}{2} \pi$.

As before in fig. 4, $\mathrm{XU}$ and $\mathrm{YU}$ are quadrants, $\mathrm{QY}=\mathrm{Q}_{1} \mathrm{X}=p$ suppose, $\mathrm{Q}_{1} \mathrm{Y}:=\mathrm{QX}=q$ suppose ;

$$
\mathrm{DX}=\mathrm{YV}, \mathrm{DX}+\mathrm{AY}=\mathrm{AV}=\mathrm{DW}=c^{\prime} .
$$

7. The angle $A D X=\phi+\psi$ in fig. 5 , as in fig. 3 ; $\mathrm{QDX}=\psi, \mathrm{Q}_{1} \mathrm{DX}=\phi$.

Similarly $\mathrm{DAY}=\phi^{\prime}+\psi^{\prime}, \mathrm{Q}_{1} \mathrm{AY}=\phi^{\prime}, \mathrm{QAY}=\psi^{\prime}$.

Then in the spherical triangle $A Q V$ in fig. 5,

(1) $\sin \mathrm{QV}=\sin \mathrm{DQ}_{\mathrm{l}}=\kappa \sin \psi^{\prime}, \sin \mathrm{AQ}=\kappa \sin \phi$,

(2) $\frac{\sin Q V}{\sin Q A V}=\kappa=\frac{\sin A Q}{\sin Q V A}=\frac{\kappa \sin \phi}{\sin Q V A}=\frac{\sin A V}{\sin A Q V}$,

so that $\mathrm{QVA}=\phi$; this is seen also from the equality of the triangles $\mathrm{QYV}, \mathrm{Q}_{1} \mathrm{DX}$, in which $\mathrm{QY}=\mathrm{Q}_{1} \mathrm{X}, \mathrm{YV}=\mathrm{DX}$, so that $\mathrm{QVY}=\mathrm{Q}_{1} \mathrm{DX}=\phi$; this is the equivalent of $\mathrm{Mr}$. RoseInnes's theorem (III.).

Also

(3) $\sin \mathrm{AQV}=\frac{\sin e^{\prime}}{\kappa}, \sin \mathrm{AQV}=\sin \mathrm{MQV}=-\cos \mathrm{DQV}$, since DQM is a right angle; and so we put, as in (8) $\$ 8$,

(4) $\mathrm{AQV}=\mathrm{am}(\mathrm{K}+w), \quad \mathrm{MQV}=\mathrm{am}(\mathrm{K}-w)$,

$$
\begin{aligned}
& \sin c^{\prime}=\kappa \operatorname{sn}(\mathrm{K}+w)=\kappa \operatorname{sn}(\mathrm{K}-w), \\
& \cos c^{\prime}=\operatorname{dn}(\mathrm{K}+w)=\operatorname{dn}(\mathrm{K}-w) .
\end{aligned}
$$


8. The addition formula of the elliptic function follows at once from a Legendre spherical triangle, in the mauner employed by Mr. Kummell ('Analyst,' 1878) ; for in the spherical triangle $\mathrm{AQV}$,

(1) $\sin p=\sin \mathrm{QY}=\sin \mathrm{AQ} \sin \mathrm{QAY}=\kappa \sin \phi \sin \psi^{\prime}$, $\cos p=\sqrt{ }\left(1-\kappa^{2} \sin ^{2} \phi \sin ^{2} \psi^{\prime}\right)$,

(2) $\cos \mathrm{AY}=\frac{\cos \mathrm{AQ}}{\cos \mathrm{QY}}=\frac{\Delta \phi}{\cos p}$,

$$
\sin \mathrm{AY}=\frac{\sin \mathrm{AQ} \cos \mathrm{QAY}}{\cos \mathrm{QY}}=\frac{\kappa \sin \phi \cos \psi^{\prime}}{\cos p}
$$

(3) $\cos Y V=\frac{\cos Q V}{\cos Q Y}=\frac{\Delta \psi^{\prime}}{\cos p}$,

$\sin \mathrm{YV}=\frac{\sin \mathrm{QV} \cos \mathrm{QVY}}{\cos \mathrm{Q} \mathrm{P}}=\frac{\kappa \sin \psi^{\prime} \cos \phi}{\cos p}$,

(4) $\cos e^{\prime}=\cos \mathrm{AV}=\cos (\mathrm{AY}+\mathrm{YV})$

$$
\begin{aligned}
& =\frac{\Delta \phi \Delta \psi^{\prime}-\kappa^{2} \sin \phi \cos \phi \sin \psi^{\prime} \cos \psi^{\prime}}{1-\kappa^{2} \sin ^{2} \phi \sin ^{2} \psi^{\prime}} \\
& =\operatorname{dn}\left(u+\mathrm{K}-u_{1}\right)=\operatorname{dn}(\mathrm{K}-w)=\operatorname{dn}(\mathrm{K}+w) .
\end{aligned}
$$

In a similar manner, with Spherical Trigonometry,

(5) $\cos A Q Y=\frac{\sin Q A Y \cos A Q}{\cos Q \bar{Y}}=\frac{\sin \psi^{\prime} \Delta \phi}{\cos p}$,

$$
\sin A Q Y=\frac{\cos Q A Y}{\cos Q Y}=\frac{\cos \psi}{\cos p},
$$

(6) $\cos \mathrm{VQY}=\frac{\sin Q V Y \cos Q V}{\cos Q Y}=\frac{\sin \phi \Delta \psi^{\prime}}{\cos p}$,

$$
\sin \mathrm{VQY}=\frac{\cos Q V Y}{\cos Q Y^{-}}=\frac{\cos \phi}{\cos \psi},
$$

(7) $\cos \mathrm{AQV}=\cos (\mathrm{AQY}+\mathrm{VQY})$

$$
\begin{aligned}
& =\frac{\sin \phi \Delta \phi \sin \psi^{\prime} \Delta \psi^{\prime}-\cos \phi \cos \psi^{\prime}}{1-\kappa^{2} \sin ^{2} \phi \sin ^{2} \psi^{\prime}} \\
& =-\operatorname{cn}\left(u+\mathrm{K}-u_{1}\right)=-\operatorname{cn}(\mathrm{K}-w)=\mathrm{cn}(\mathrm{K}+w),
\end{aligned}
$$

(8) $\mathrm{AQV}=\mathrm{am}(\mathrm{K}+w), \quad \mathrm{DQV}=\frac{1}{2} \pi+a \mathrm{~m}(\mathrm{~K}-w)$.

9. In the reciprocal diagram of fig. 4 , drawn on the left hand, $R$ is the pole of $X Y$, and the perimeter of the triangle 
$\mathrm{ARD}$ is $\pi$; the tangent intercept $\mathrm{ZZ}^{\prime}$ by the cyclic arcs is $\frac{1}{2} \pi$, so that $\mathrm{QZZ}^{\prime}$ is a spherical triangular octant; $\mathrm{RZ}=\mathrm{ZK}, \mathrm{RD}=\mathrm{DK}, \mathrm{RA}=\mathrm{AK}^{\prime}$; and if $\mathrm{R}, \mathrm{Z}$ moves to $r, z$, as $\mathrm{Q}$ advances to $q$, and $\mathrm{D} z$ crosses $\mathrm{ZR}$ in $z^{\prime}$,

(1) $\cos c d u=\cos \mathrm{DQ} d \phi=\sin \mathrm{DZ} d \phi=\mathrm{Zz}^{\prime}$

$$
=\operatorname{arc} \mathrm{R} r-r z+\mathrm{RZ} \text {, }
$$

(2) $u \cos c=\operatorname{arc} \mathrm{ER}-\mathrm{RZ}$,

thus representing the time by the difference of the arc ER and $R Z$.

In the reciprocal part of the diagram in fig. 5, where the tangents at $R, R_{1}$ intersect in $S$,

(3) $\mathrm{AS}+\mathrm{DS}=\pi-c^{\prime}$,

(4) $\left(u_{1}-u\right) \cos c=\operatorname{arc} R_{1}-R_{1} Z_{1}+R Z$;

(5) $\quad \operatorname{arc} \mathrm{RR}_{1}=\mathrm{SR}+\mathrm{SR} \mathrm{R}_{1}-$ constant (Salmon, $\S 252$ ),

(6) $\left(u_{1}-u\right) \cos c+a \operatorname{constant}=\mathrm{SZ}-\mathrm{SZ}_{1}=\mathrm{SZ}+\mathrm{SZ}_{1}{ }^{\prime}-\frac{1}{2} \pi$.

The Spherical Trigonometry interpretation is the same as before in $\S 8$; since

(7) $q=\mathrm{XQ}=\mathrm{XSQ}=\frac{1}{2} \pi-\mathrm{DSZ}=\frac{1}{2} \pi-\mathrm{ASZ}_{1}^{\prime}$,

$\sin q=\sin \mathrm{DQ} \sin \mathrm{QDX}=\kappa \sin \phi^{\prime} \sin \psi$,

(8) $\cos \mathrm{SZ}=\frac{\cos \mathrm{ZDS}}{\sin \mathrm{DSZ}}=\frac{\cos \psi}{\cos q}$,

$$
\sin \mathrm{SZ}=\frac{\sin \mathrm{ZDS} \sin \mathrm{DZ}}{\sin \mathrm{DSZ}}=\frac{\sin \psi \Delta \phi^{\prime}}{\cos q}
$$

(9) $\cos \mathrm{SZ}_{1}^{\prime}=\frac{\cos \mathrm{Z}_{1}^{\prime} \mathrm{AS}}{\sin \mathrm{AS} Z_{1}^{\prime}}=\frac{\cos \phi^{\prime}}{\cos q}$,

$$
\sin \mathrm{SZ}_{1}^{\prime}=\frac{\sin \mathrm{Z}_{1}^{\prime} \mathrm{AS} \sin \mathrm{AZ}_{1}^{\prime}}{\sin \mathrm{AS} \mathrm{Z}_{1}^{\prime}}=\frac{\sin \phi^{\prime} \Delta \psi}{\cos q},
$$

(10) $\sin \left(\mathrm{SZ}+\mathrm{S} Z_{1}^{\prime}\right)=\frac{\sin \psi \cos \phi^{\prime} \Delta \phi^{\prime}+\sin \phi^{\prime} \cos \psi \Delta \psi}{\cos ^{2} q}$

$$
\begin{aligned}
& \quad \operatorname{sn}\left(\mathrm{K}-u+u_{1}\right)=\operatorname{sn}(\mathrm{K}+w), \\
& \mathrm{SZ}+\mathrm{SZ}_{1}{ }^{\prime}=\mathrm{am}(\mathrm{K}+w) .
\end{aligned}
$$


And with

$$
\begin{aligned}
\text { (11) } p=\mathrm{XQ}_{1} & =\mathrm{XSQ}_{1}=\frac{1}{2} \pi-\mathrm{DSZ}_{1}=\frac{1}{2} \pi-\mathrm{ASZ}^{\prime}, \\
\text { (12) } \sin \mathrm{SZ} & =\cos \mathrm{SZ}^{\prime}=\frac{\cos \mathrm{SAZ}}{\sin \mathrm{ASZ}}=\frac{\cos \psi^{\prime}}{\cos p}, \\
\cos \mathrm{SZ} & =\frac{\sin \mathrm{SAZ} \sin \mathrm{AZ}^{\prime}}{\sin \mathrm{ASZ} Z^{\prime}}=\frac{\sin \psi^{\prime} \Delta \phi}{\cos p}, \\
\text { (13) } \cos \mathrm{SZ}_{1} & =\frac{\cos \mathrm{SDZ}_{1}}{\sin \mathrm{DSZ} Z_{1}}=\frac{\cos \phi}{\cos p}, \\
\sin \mathrm{SZ}_{1} & =\frac{\sin \mathrm{SDZ}_{1} \sin \mathrm{DZ}_{1}}{\sin \mathrm{DSZ}_{1}}=\frac{\sin \phi \Delta \psi^{\prime}}{\cos p},
\end{aligned}
$$

$$
\begin{aligned}
\sin \left(\mathrm{SZ}-\mathrm{SZ}_{1}\right) & =\frac{\cos \phi \cos \psi^{\prime}-\sin \phi \sin \psi^{\prime} \Delta \phi \Delta \psi^{\prime}}{\cos ^{2} p} \\
& =\operatorname{cn}\left(u+\mathrm{K}-u_{1}\right)=\operatorname{cn}(\mathrm{K}-w),
\end{aligned}
$$

(15) $\mathrm{SZ}-\mathrm{SZ}_{1}=\frac{1}{2} \pi-\mathrm{am}(\mathrm{K}-w)$,

$$
\mathrm{Z}_{1} \mathrm{Z}_{1}{ }^{\prime}=\frac{1}{2} \pi=\mathrm{am}(\mathrm{K}+w)+\mathrm{am}(\mathrm{K}-w)-\frac{1}{2} \pi,
$$

(16) $\mathrm{SZ}+\mathrm{SZ}_{1}^{\prime}=\operatorname{am}(\mathrm{K}+w)$.

Thus the constant in (6) is am $(\mathrm{K}+w)-\frac{1}{2} \pi-\kappa^{\prime} w$.

The sphero-conic ERE' of fig. 4 is the projection on the sphere of the polar reciprocal of the circle AQD of fig. 1 with respect to $D$, and this is the parabola $E R$; while the sphero-conic $\mathrm{S}$ in fig. 5 will be the projection of the hyperbola of $\mathrm{S}$ in fig. 3, polar reciprocal of the circle $a \mathrm{~T} d$.

10. The motion of $\mathrm{P}$ at the same level as $\mathrm{Q}$ in fig. 1 . oscillating on the arc $\mathrm{BAB}^{\prime}$ of the circle on the diameter $\mathrm{AE}$, will represent the associated motion of a pendulum, swinging through an angle $4 c$, and then if $\theta$ denotes the inclination of the pendulum $O P$ to the vertical

(1) $\sin ^{2} \frac{1}{2} \theta=\frac{\mathrm{AN}}{\mathrm{AE}}=\frac{\mathrm{AD}}{\mathrm{AE}} \cdot \frac{\mathrm{AN}}{\mathrm{AD}}=\kappa^{2} \sin ^{2} \phi$,

$$
\cos \frac{1}{2} \theta=\Delta \phi=\cos \mathrm{DQ}, \quad \frac{1}{2} \theta=\mathrm{DQ}, \quad \text { in fig. } 4 .
$$

Draw a circle through $\mathrm{B}$ and $\mathrm{B}^{\prime}$, centre $o$, in fig. 6; draw $\mathrm{PB}$, and $\mathrm{P}_{p} \mathrm{~B}^{\prime}$ crossing this circle at $p$; and draw $\mathrm{PH}$ and $\mathrm{O} q$ perpendicular to $\mathrm{BB}^{\prime}$ and $\mathrm{PB}^{\prime}$.

Phil. Mag. S. 6. Vol. 20. No. 118. Oct. 1910. 3 C 
The triangles $\mathrm{P} p \mathrm{~B}$ and $\mathrm{OoB}$ are similar, and so are the triangles $\mathrm{PHB}, \mathrm{P} q \mathrm{O}$; so that

$$
\frac{\mathrm{P} p}{\mathrm{O} o}=\frac{\mathrm{PB}}{\overline{\mathrm{OB}}}=\frac{\mathrm{PB}}{\mathrm{PO}}=\frac{\mathrm{PH}}{\overline{\mathrm{P}} q}=\frac{2 \mathrm{PH}}{\overline{\mathrm{P}} \overline{\mathrm{B}}^{\prime}},
$$

and if $\mathrm{PRP}_{1}$ is tangent to this circle,

(3) $\mathrm{PR}^{2}=\mathrm{P} p \cdot \mathrm{PB}^{\prime}=2 \mathrm{O} o . \mathrm{PH}, \mathrm{P}_{1} \mathrm{R}^{2}=2 \mathrm{O}$ o. $\mathrm{P}_{1} \mathrm{H}_{1}$.

Fig. 6.

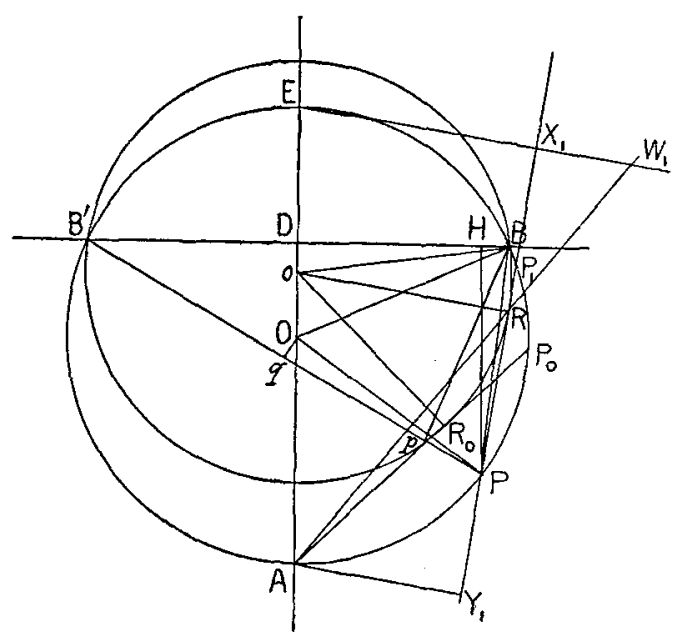

If $P$ and $P_{1}$ are particles os cillating with the velocity due to the level $\mathrm{BB}^{\prime}$,

$$
\frac{\text { vel. of } \mathrm{P}}{\text { vel. of } \mathrm{P}_{1}}=\sqrt{\frac{\mathrm{PH}}{\mathrm{P}_{1} \mathrm{H}_{1}}}=\frac{\mathrm{PR}}{\mathrm{P}_{1} \mathrm{R}} \text {, }
$$

so that, as before in $\S 4, \mathrm{PP}_{1}$ will continue to touch the circle, centre $o$, during the subsequent motion; and the centre $o$ has been chosen so as to bring $\mathrm{P}_{1}$ to the same level as $Q_{1}$, and then $P Q, P_{1} Q_{1}$ will continue to be horizontal, by a suitable arrangement of gravity.

11. It is desirable to have a geometrical interpretation of the addition formula of (4) and (7), $\S 8$, on the plane in figs. 3 and 6 ; this is seen by drawing AT, AR to meet DX and $\mathrm{EX}_{1}$ produced, drawn perpendicular to $\mathrm{QQ}_{1}$ and $P P_{1}$, in $\mathrm{W}$ and $\mathrm{W}_{1}$. 
Then if $\mathrm{P}_{1}$ and $\mathrm{Q}_{1}$ come to $\mathrm{P}_{0}$ and $\mathrm{Q}_{0}$ when $\mathrm{P}$ and $\mathrm{Q}$ are starting together from $A$, when

$u=0, u_{1}=w$, and $\mathrm{ADQ}_{0}=\gamma=\mathrm{am} w, \quad \cos \mathrm{AEP}_{0}=\Delta \gamma$,

(1) $\frac{\mathrm{DW}}{\mathrm{DA}}=\frac{c \mathrm{~T}}{c \mathrm{~A}}=\frac{c \mathrm{~T}_{0}}{c \mathrm{~A}}=\frac{\mathrm{DQ}_{0}}{\mathrm{DA}}=\cos \mathrm{ADQ}_{0}=\cos \gamma, \quad \mathrm{DW}=\mathrm{DQ}_{0}$;

(2) $\frac{\mathrm{EW}_{1}}{\mathrm{EA}}=\frac{o \mathrm{R}}{o \mathrm{~A}}=\frac{o \mathrm{R}_{0}}{o \mathrm{~A}}=\frac{\mathrm{EP}_{0}}{\mathrm{EA}}=\cos \mathrm{AEP}_{0}=\Delta \gamma, \quad \mathrm{EW}_{1}=\mathrm{EP}_{0}$.

(3) $\mathrm{AQ}=\mathrm{AD} \sin \phi, \quad \mathrm{DQ}=\mathrm{AD} \cos \phi$,

$\mathrm{AP}=\mathrm{AE} . \kappa \sin \phi=\mathrm{AB} \sin \phi, \quad \mathrm{EP}=\mathrm{AE} . \Delta \phi$,

so that, by Euclid VI. C,

(4) $\mathrm{DX}=\frac{\mathrm{DQ} \cdot \mathrm{DQ}}{\mathrm{AD}}=\mathrm{AD} \cos \phi \cos \psi$,

$\mathrm{AY}=\frac{\mathrm{AQ} \cdot \mathrm{AQ}}{\mathrm{AD}}=\mathrm{AD} \sin \phi \sin \psi$,

(5) $\mathrm{AY}_{1}=\frac{\mathrm{AP} \cdot \mathrm{AP}_{1}}{\mathrm{AE}}=\mathrm{AE} \cdot \kappa^{2} \sin \phi \sin \psi$

$=\mathrm{AD} \sin \phi \sin \psi=\mathrm{AY}$,

$\mathrm{EX}_{1}=\frac{\mathrm{EP} \cdot \mathrm{EP}_{1}}{\mathrm{AE}}=\mathrm{AE} \cdot \Delta \phi \Delta \psi$.

(6) $\quad \cos \gamma=\frac{\mathrm{DW}}{\mathrm{AD}}=\frac{\mathrm{DX}}{\mathrm{AD}}+\frac{\mathrm{XW}}{\mathrm{AD}}=\frac{\mathrm{DX}}{\mathrm{AD}}+\frac{\mathrm{AY}}{\mathrm{AD}} \cdot \frac{\mathrm{XW}}{\mathrm{AY}}$,

$\frac{\mathrm{XW}}{\mathrm{AY}}=\frac{\mathrm{XT}}{\mathrm{TY}}=\frac{\mathrm{D} c}{c \mathrm{~A}}=\frac{\mathrm{Q}_{0} \mathrm{~T}_{0}}{\mathrm{~T}_{0} \mathrm{~A}}=\frac{\mathrm{Q}_{0} \mathrm{~L}}{\mathrm{LA}}=\Delta \gamma$,

(7) $\cos \gamma=\cos \phi \cos \psi+\sin \phi \sin \psi \Delta \gamma$.

(8) $\Delta \gamma=\frac{\mathrm{EW}_{1}}{\mathrm{AE}}=\frac{\mathrm{EX}_{1}}{\mathrm{AE}}+\frac{\mathrm{X}_{1} \mathrm{~W}_{1}}{\mathrm{AE}}=\frac{\mathrm{EX}_{1}}{\mathrm{AE}}+\frac{\mathrm{AY}_{1}}{\mathrm{AE}} \cdot \frac{\mathrm{X}_{1} \mathrm{~W}_{1}}{\mathrm{A \textrm {Y } _ { 1 }}}$,

(9) $\frac{\mathrm{X}_{1} \mathrm{~W}_{1}}{\mathrm{AY}_{1}}=\frac{\mathrm{X}_{1} \mathrm{R}}{\mathrm{RY_{1 }}}=\frac{\mathrm{E} o}{o \mathrm{~A}}=\frac{\mathrm{P}_{0} \mathrm{R}_{0}}{\mathrm{R}_{0} \mathrm{~A}}=\sqrt{\frac{\mathrm{DN}_{0}}{\mathrm{DA}}}=\cos \gamma$,

(10) $\Delta \gamma=\Delta \phi \Delta \psi+\kappa^{2} \sin \phi \sin \psi \cos \gamma$.

Here (7) and (10) are the well-known formulas of Legendre; and thence, as before in ( 7$)$ and (4), $\S 8$,

(11) $\cos \gamma=\cos \phi \cos \psi+\sin \phi \sin \psi\left(\Delta \phi \Delta \psi+\kappa^{2} \sin \phi \sin \psi \cos \gamma\right)$ $\cos \gamma=\frac{\cos \phi \cos \psi+\sin \phi \sin \psi \Delta \phi \Delta \psi}{1-\kappa^{2} \sin ^{2} \phi \sin ^{2} \psi} ;$

(12) $\Delta \gamma=\Delta \phi \Delta \psi+\kappa^{2} \sin \phi \sin \psi(\cos \phi \cos \psi+\sin \phi \sin \psi \Delta \gamma)$, $\Delta \gamma=\frac{\Delta \phi \Delta \psi+\kappa^{2} \sin \phi \sin \psi \cos \phi \cos \psi}{1-\kappa^{2} \sin ^{2} \phi \sin ^{2} \psi}$. 
Or, geometrically,

$$
\begin{aligned}
& \frac{\cos \gamma-\cos \phi \cos \psi}{\sin \phi \sin \psi}=\frac{\mathrm{XW}}{\mathrm{AY}}=\frac{\mathrm{EW}}{\mathrm{A} \overline{\mathrm{E}}}=\frac{\mathrm{EX}}{\mathrm{A} \bar{E}}+\frac{\mathrm{X}_{1} \mathrm{~W}_{1}}{\mathrm{AE}} \\
& =\Delta \phi \Delta \psi+\kappa^{2} \sin \phi \sin \psi \cos \gamma \\
& \begin{aligned}
\frac{\Delta \gamma-\Delta \phi \Delta \psi}{\kappa^{2} \sin \phi \sin \psi}=\frac{\mathrm{X}_{1} W_{1}}{\mathrm{~A} \bar{W}_{1}} & =\frac{\mathrm{DW}}{\mathrm{AD}}=\frac{\mathrm{DX}}{\mathrm{AD}}+\frac{\mathrm{XW}}{\mathrm{AD}} \\
& =\cos \phi \cos \psi+\sin \phi \sin \psi \Delta \gamma,
\end{aligned}
\end{aligned}
$$

equivalent to (11) and (12) above.

So also the formula for $\sin \gamma=\operatorname{sn} w$ can be interpreted.

LXXXII. The Optical Determination of Stress. By E. G. Coк Er, M.A., D.Sc., Professor of Mechanical Engineering, City and Giulds of London Technical College, Finsbury**

7 THE principal advances in our experimental knowledge of the strength and properties of materials have been made by the use of mechanical apparatus for applying stress and measuring strain, and instruments of this class are now in general use possessing a sufficient degree of accuracy for the most refined measurements, but whatever applications purely mechanical methods may have, they possess a common characteristic feature that measurements must be taken over a definite length, area, or volume maintained in a standard condition throughout in order that the state of stress or strain may be referred to some standard measure possessed by the instrument, or by which it is calibrated. Whatever the arrangement may be, it is not in general possible to measure the stress or strain at a point, if the body is subjected to stress varying from point to point.

This defect in purely mechanical devices is one which from the nature of the case is hardly likely to be overcome entirely, yet in the great majority of the problems which arise in practice the stresses change very rapidly from point to point, and experimental information, if it exists, has almost invariably been obtained by using mechanical apparatus incapable of determining the stress at a point. Mathematical researches of the state of stress and strain in bodies give exact solutions of a variely of complicated problems; but some of the simplest forms of practical construction offer problems of the greatest difficulty, as for eximple the determination of the stresses in hooks, chain links, and rivotted

* Communicated by the Author: read in abstract at the British Association, Sheffield. 